\title{
Knowledge, Perceived Competency and Problems in Using Basic Periodontal Examination (BPE) as a Screening Tool for Periodontal Patients by New Clinical Dental Students
}

\author{
Mahyunah Masud ${ }^{1 *}$ \\ ${ }^{1}$ Faculty of Dentistry, Universiti Teknologi MARA (UiTM), Malaysia \\ *Corresponding Author: Mahyunah Masud, Faculty of Dentistry, Universiti Teknologi MARA (UiTM), \\ Malaysia; Email: mahyunah@uitm.edu.my
}

Received Date: 23-06-2021; Accepted Date: 23-07-2021; Published Date: 30-07-2021

Copyright $^{\odot} 2021$ by Masud M. All rights reserved. This is an open access article distributed under the terms of the Creative Commons Attribution License, which permits unrestricted use, distribution and reproduction in any medium, provided the original author and source are credited.

\begin{abstract}
Background: Recognition of disease is an essential component in periodontal disease management. It requires effective Basic Periodontal Examination (BPE), to guide the need for treatment. This study aimed to assess the year 3 clinical students' knowledge, usage, perceived competency, and problems in conducting BPE. It also assessed the effectiveness of the teaching and learning ( $\mathrm{T}$ and $\mathrm{L})$ of BPE.
\end{abstract}

Method: The cross-sectional, self-administered questionnaire survey was conducted among the year 3 students in two dental institutions. Lectures and hands-on training given to the students were followed by five months of clinical exposure on BPE to screen new patients. The thirteen questions provided to the students were divided into demographic data, number of BPE conducted, time taken, perception on knowledge on BPE, usage, competency and problems that they might have faced while conducting BPE.

Results: A total of eighty-five year 3 students participated in this study with 422 BPE performance recorded. The average time taken was 5 to 15 minutes per patient. On perceived knowledge and clinical performance, $58 \%$ presented with moderate to high score on the understanding, indication, codes, instruments used and skill on BPE. Most perceived BPE as a useful guide for treatment needs but the main problems were uncooperative patients and too many sites to probe. 
Conclusion: Although new clinical students perceived BPE was useful and 50\% felt that they were competent, only $20 \%$ of them had high knowledge and skill. Effective $\mathrm{T}$ and L with appropriate exposure to BPE were needed to improve during the next two years of study before graduation.

\section{Keywords}

Basic Periodontal Examination (BPE); Screening Tool; Knowledge; Perceived Competency

\section{Introduction}

Periodontal diseases especially periodontitis represent a major global disease burden with devastating effects on oral health, a close link with general health and account for huge socioeconomic impacts and massive health care costs worldwide [1]. In Malaysia, 94\% of the population suffered from periodontal diseases at all levels of severity, with $18.2 \%$ in severe condition [2-3].

The management of periodontal diseases can be difficult if a clinician lacks skills in recognizing its signs. The gingiva may appear to be healthy although the underlying condition indicates otherwise. Furthermore, patients may not complain of pain unless the disease is severe and longstanding or when the teeth are too loose in the mouth. Recognition of signs of disease is an essential component in periodontal disease management. It requires a good understanding of the basis of management as it will highlight the needs of treatment and ultimately determines the treatment outcome. Before a diagnosis and comprehensive treatment planning can be made, appropriate examination and investigation must be carried out. A basic periodontal examination should include a direct visualization for gingival condition, history/signs of disease experience, manual probing of the periodontal sulcus or pocket depths.

A study on the curriculum for primary care dentistry recommended curricular emphasis initially placed on the ability to diagnose and expanded on competency in dental subjects including periodontics and the $\mathrm{T}$ and $\mathrm{L}$ of periodontology involve students acquiring basic skills in assessing the condition of teeth and supporting structures, making a diagnosis, the summary of findings and highlighting the needs of treatment before being able to carry out the appropriate treatment modality on their patients [4]. Community Periodontal Index (CPI) was suggested in the earlier years for screening of periodontal patients [5]. This was the modification of the Community Periodontal Index of Treatment Needs (CPITN) whereby the treatment needs component was eliminated [6-8]. Other screening tools were periodontal screening and recording and later BPE $[9,10]$. Despite the difference in their names and 
protocols, these procedures aim at assessing the periodontal condition during patients' screening and facilitate management planning during the first visit.

BPE status during new patient screening has the advantage of giving clinicians some indications of the overall periodontal condition. In addition, it provides a prompt guide of what could be done for the patient. It is a relatively simple method, requires only a short learning time and the use of basic instruments in a simple dental examination. All students were required to perform BPE on all their patients except on children. They should understand why BPE is done, the instruments used, BPE codes and the treatment recommended for each code. Thus, this study aimed to assess the year clinical students' knowledge, usage, perceived competency and problems in conducting BPE. It also assessed the effectiveness of the teaching and learning (T and L) of BPE.

\section{Materials and Methods}

This cross-sectional study was carried out in two dental schools in the Klang Valley in Malaysia and involved the year 3 students. At year 2 they were given a lecture on BPE and at the beginning of year 3 , they were taught through a 45-minutes video, a hands-on demonstration on the principles of BPE including armamentarium, sitting position, and instrumentation technique in their respective schools using the same teaching manual. Following two weeks of 3-hours practical sessions/week on teeth models mounted on phantom heads in simulation clinics, students were then assessed on their knowledge and skills in conducting BPE on the models. Once they have fulfilled the basic requirements and showed satisfactory competency, students performed BPE intraorally under the supervision of the clinical trainers.

After approximately five months of clinical exposure in screening new patients in the student's clinic, they were asked to complete a self-administered questionnaire consisted of thirteen questions in three main parts namely students' clinical usage of BPE, their perceived understanding of the concept of BPE, clinical competency, and problems that they might have faced while conducting BPE in their clinical practice. Finally, a simple quiz was given to the students to assess their knowledge of BPE. Questions were set on the objectives of BPE, instruments, and codes used, indication for use, and limitations of the procedure. All correct answers were added for each student and total scores were divided into three categories for students' tested knowledge: low (scores 11-14), moderate (score 8-10) and high level of knowledge (score 5-7). All responses and scores were entered and analyzed in the Statistical Package for Social Science (SPSS) computer software version 15. 


\section{Results}

A total of eighty-five (85) Year 3 dental students in the two universities participated in this study. There were $65.9 \%(n=56)$ students from Universiti Kebangsaan Malaysia (UKM) and $34.1 \%(n=29)$ from Universiti Teknologi MARA (UiTM). Throughout the studied teaching and learning ( $\mathrm{T}$ and $\mathrm{L}$ ) period, students have performed a total of 422 BPEs on their patients, ranging from 1 to 11 experiences per student and an average of 5 BPE per student. Majority (83.5\%; $\mathrm{n}=71$ ) of them conducted 3-7 BPEs each (Fig. 1). The average time taken for most of the students $(83.5 \%, \mathrm{n}=71)$ to do BPE was 5 to 15 minutes per patient (Fig. 2).

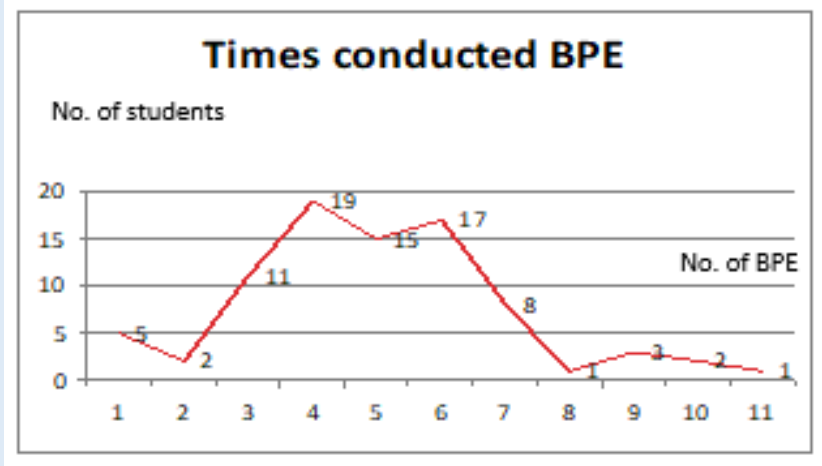

Figure 1: Numbers of times conducted BPE.

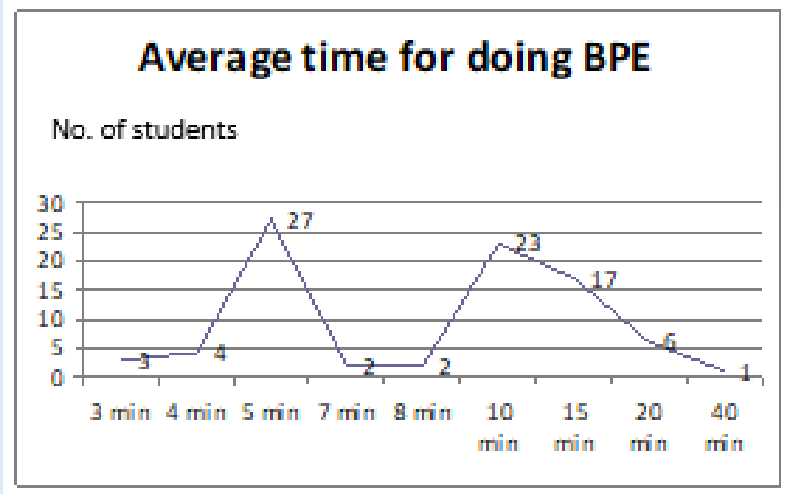

Figure 2: Average time for doing BPE.

\begin{tabular}{|c|c|c|c|}
\hline Students Perceived & Low (\%) (n) & Moderate (\%) (n) & High (\%) (n) \\
\hline Knowledge of BPE & $(42)(36)$ & $(38)(32)$ & $(20)(17)$ \\
\hline
\end{tabular}

Table 1: Students' perception on knowledge of BPE.

On the tested knowledge on theory and clinical performance, the quiz revealed $38 \%$ and $20 \%$ scored moderate to high respectively on the understanding, indication, codes, instruments used and skill on BPE. 
Ninety-four percent $(n=80)$ of the students perceived that BPE is useful in their clinical practice, while 5\% $(n=5)$ found that they did not benefit from the use of BPE when examining their patients. $51 \%(\mathrm{n}=43)$ perceived that they were competent in conducting BPE, $18 \%(\mathrm{n}=15)$ were not competent and $32.8 \%(\mathrm{n}=27)$ did not seem to know their competency in BPE. Most students $(69.4 \%)$ perceived that the newly acquired knowledge was not difficult to conduct while $21.1 \%(n=18)$ felt that the procedure was difficult to do (Fig. 3-5).

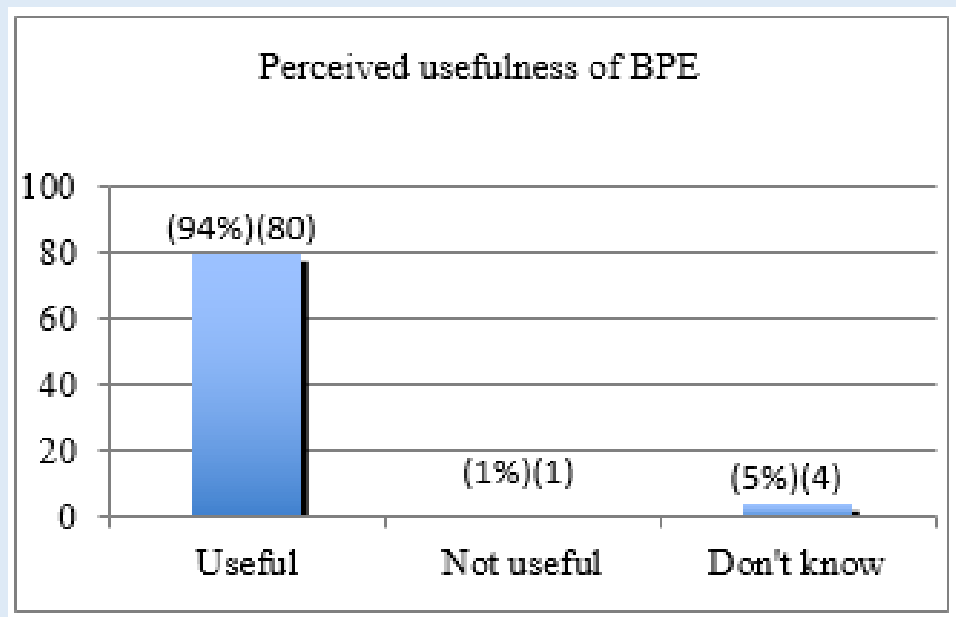

Figure 3: Perceived usefulness of BPE.

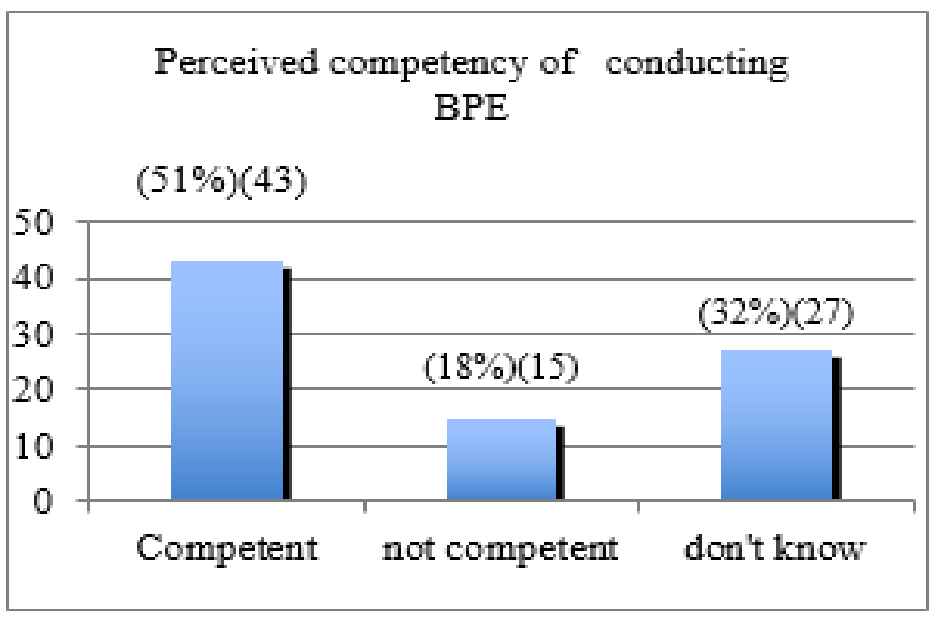

Figure 4: Perceived competency conducting BPE. 


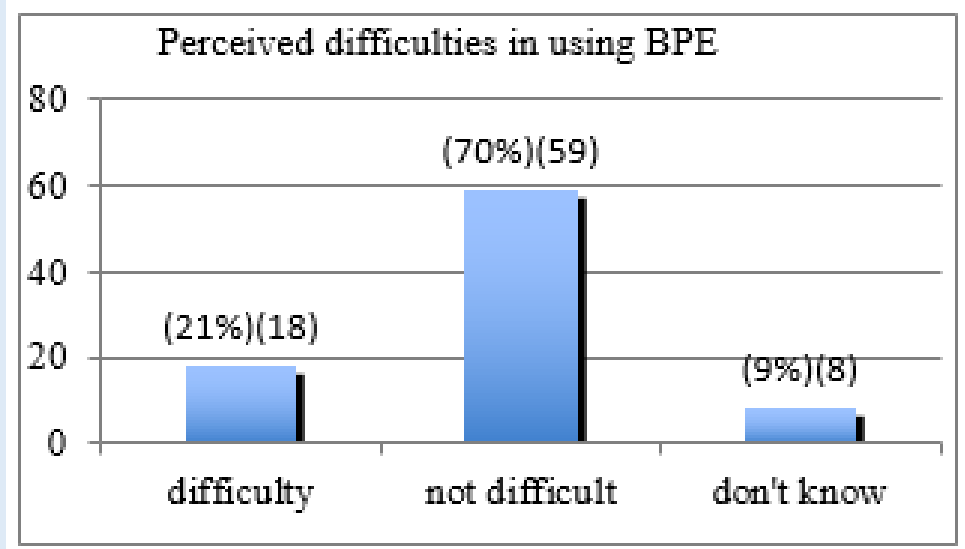

Figure 5: Perceived difficulties in using BPE.

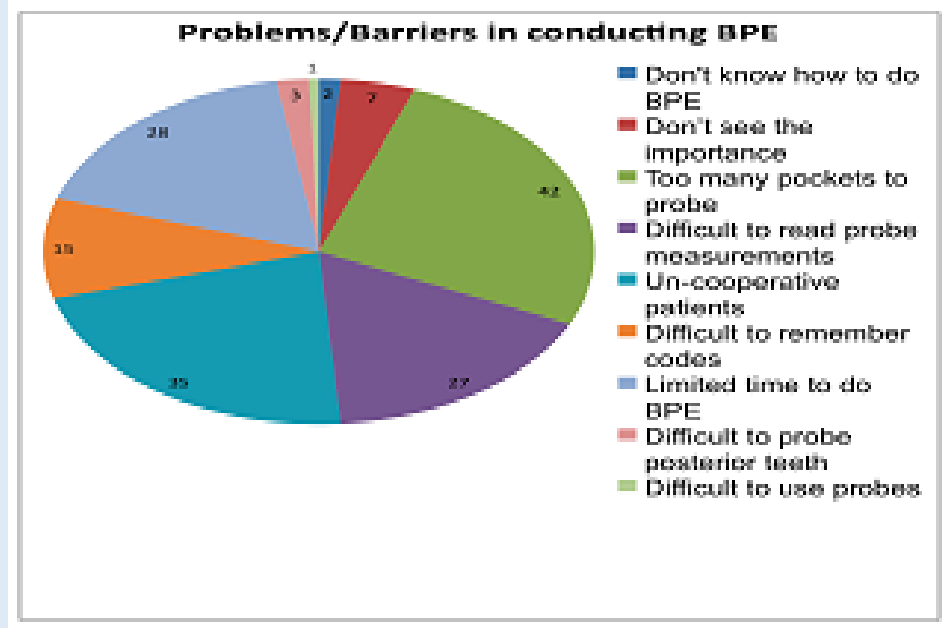

Figure 6: Problem/barriers in conducting BPE.

Among the common problems faced by students while doing BPE were too many sites to probe (49\%), uncooperative patients $(41 \%, \mathrm{n}=35)$, limited time to do BPE $(33 \%, \mathrm{n}=28)$, and difficult to read markings on the probe for measurements of pocket depth (32\%), sadly 7 (0.8\%) students did not see the importance of BPE (Fig. 6) as a screening tool.

\section{Discussion}

Recognition of signs and symptoms of the disease is an integral component in the management of oral disease particularly periodontal disease. Before a comprehensive treatment can be carried out, relevant examination and reliable investigation must be done and fast enough to identify any problems that may be present. If any of these problems are detected, the clinician must decide whether he/she will treat the patient or refer the patient to a specialist. The 
prevention, detection, and treatment of periodontal disease should be recognized early and can therefore be dealt with accordingly [11]. Throughout the study period of about six (6) months, each student conducted an average of 5 BPE, taking an average time of 5-15 minutes per patient. It was as expected, for newly exposed procedures in the clinical management of patients. However, with just over five months period, $58 \%$ of students scored moderate to high knowledge on the understanding, indication, codes, instruments used and skill on BPE. Thus, BPE is a relatively simple method, requires only a short learning time and uses few basic instruments in the examination of patients [12].

In countries such as Australia and Britain, the basic periodontal examination for screening of periodontal disease had been made compulsory as a minimal requirement for the parameter of care to ensure high-quality dental management and treatment outcome. Feedbacks from participating general dental practitioners indicated that $80-91 \%$ of them screened all new patients for periodontal disease [13]. A study by Tugnait, et al., in 2004, reported that $91 \%$ of dentists used BPE in new patients as clinical screening and in addition to the increasing concern on the progress of periodontal disease and the current link of the disease with several systemic conditions and chronic disease [14-16]. An educational model and clinical materials were developed which aimed at alerting patients, dental and medical personnel to the increased risk for systemic health problems when inflammatory periodontal disease is present [17]. Dental clinicians especially newly graduated dental professionals must be well informed and competent in at least diagnosing the signs of disease in the general population. The study has also shown that the more experienced the clinician, the more accurate their diagnoses were [18]. The new curriculum review for dental undergraduate programs in our universities involved, highlighted this skill as an essential learning outcome for clinical dental students. In this study, a teaching-learning package was developed, used together in both schools and taught before the students formally entered the treatment clinics. After almost half a years' exposure in the clinics, many of the students have had experience in conducted BPE on their new patients. Although the length of time used for doing BPE varies among the students, the range between 5-15 minutes is acceptable for this batch, considering the amount of experience they had throughout the first year of clinical studies.

The majority of the year 3 students perceived that BPE is useful in their clinical practice, $51 \%$ perceived that they were competent in conducting BPE and $69.4 \%$ perceived that the newly acquired knowledge was not difficult to conduct. In comparison to the study done on year 5 students doing BPE, they presented a significant difference in the understanding of the clinical implementation of the BPE in the final months of education. Interestingly, BPE in theory and BPE in practice would have shown differences in the theoretical knowledge of each student. They were confident in their competency despite high inaccuracy rates in BPE code identification indicating that reskilling before graduating be appropriate for the students [19]. Our year 3 faced problems with too many sites to probe, uncooperative patients and time Masud M | Volume 2; Issue 2 (2021) | JDHOR-2(2)-030 | Research Article 
limitations, however, they would have two years more to perfect their BPE skill and be competent enough on graduation as they would be doing BPE as a screening tool for another four semesters not just for periodontal patients but for all patients that they would manage.

\section{Conclusion}

BPE is an integral component of periodontal management. The new year 3 clinical dental students perceived being competent in conducting the BPE on patients after appropriate training. Although the students showed good competency immediately after the initial training in simulation clinics, a more effective teaching and learning approach with sufficient clinical exposure and continuous assessment on the theory are needed to improve their clinical knowledge and skills. Concurring with further clinical learning sessions scheduled for years 4 and 5, emphasis on this curriculum objective can be implemented and achieved.

\section{Recommendation}

As a guide, for all patients attending the students' clinic with BPE 1 and 2, they may proceed with motivation, oral hygiene instruction, scaling and supportive periodontal therapy. While those with BPE 3 and 4, the patient needed to be examined further and placed under comprehensive periodontal care. Suggestion has also been forwarded to all practicing dentists in the public service to practice BPE as a screening tool for all patients.

\section{Acknowledgment}

The author wished to thank the Deans of Faculty of Dentistry, UiTM in respective schools for their support in this dental education study.

\section{Conflict of Interest Disclosure}

There is no conflict of interest and no funding sources supporting the study.

\section{References}

1. FDI world dental federation, global periodontal health. Adopted by the FDI general assembly. Int Dent J. 2019;69:1.

2. National Oral Health Survey of Adults in Malaysia. 2010.

3. Mohd Dom TN, Ayob R, Abd Muttalib K. Aljunid SM. The national economic burden associated with management of periodontitis in Malaysia. Int J Dent. 2016:1891074.

4. Rovin S. A curriculum for primary care dentistry. J Dent Educ. 1977;41(4):176-90.

5. Wilherm K. Community Periodontal Index (CPI) - encyclopaedia of public health. 2008.

Masud M | Volume 2; Issue 2 (2021) | JDHOR-2(2)-030 | Research Article 
6. Ainamo J, Barmes D, Beagrie G, Cutress T, Martin J, Sardo-InInfirri J. Development of the World Health Organisation (WHO) Community Periodontal Index of Treatment Needs (CPITN). Int Den J. 1982;32(3):281-91.

7. Gjermo P. CPITN as a basic periodontal examination in dental practice. Int Dental J 1994;5(1): 547-52.

8. Barmes D. CPITN-WHO initiatives. Int Dent J. 1994;44(5):523-5.

9. Nasi JH. Background to and implementation of the Periodontal Screening and Recording (PSR) procedure in USA. Int Dent J. 1994;44(1):585-8.

10. Periodontology in general dental practice in the United Kingdom: a policy statement. Br Soc Periodontol. 2001.

11. Stone JS. Abbreviated periodontal examination J Am Dent Assoc. 1976;92(1):140-4.

12. Basic periodontal examination: A policy statement - Br Soc Periodontol. 2016. [Last accessed on July 22 , 2021] www.bsperio.org

13. Dowell P, Chapple IL. British society of periodontology referral policy and parameters of care. Dental Update. 2002;29(7):352-3.

14. Tugnait A, Clerehugh V, Hirschmann PN. Use of the basic periodontal examination and radiographs in the assessment of periodontal diseases in general dental practice: J Dent. 2004;32(1):17-25.

15. Darby IB, Angkasa F, Duong C, Ho D, Legudi S, Pham K, et al. Factors influencing the diagnosis and treatment of periodontal disease by dental practitioners in Victoria. Aust Den J. 2005;50(1):37-41.

16. Wilder RS, Thomas KM, Jared H. Periodontal - systemic disease education in United States dental hygienist program: J Dent Edu. 2008;72(6):669-79.

17. Bush BC, Donley TG, A model for dental hygiene education concerning the relationship between periodontal health and systemic health: Edu Health. 2002:15(1):19-26.

18. Eberhard J, Klomp HJ, Foge M, Hedderich J, Schmidt HG. The intermediate effect and diagnostic accuracy in clinical case recall of students and experts in dental medicine. Eur J Dent Educ. 2009;13(3):128-34.

19. Fornshaw E, Taylor J. The understanding of the basic periodontal examination amongst final undergraduate students; a survey. Br Dent Assoc. 2021:40-5. 